\title{
Skin-sparing mastectomy with immediate nipple reconstruction during autologous latissimus dorsi breast reconstruction: A review of patient satisfaction
}

\author{
Ciaran M Hurley, Adrian McArdle, Kenneth M Joyce, Eoin O’Broin \\ Department of Plastic and Reconstructive Surgery, Cork University Hospital, Cork, Ireland
}

Background Nipple-areolar complex (NAC) reconstruction following curative mastectomy is traditionally performed as a second-stage procedure several months after initial breast reconstruction. The recent literature has documented the increasing popularity of immediate nipple reconstruction carried out simultaneously during autologous reconstruction. The aim of this study was to evaluate the surgical outcomes and patient satisfaction with immediate breast and nipple reconstruction performed in a single stage after skin-sparing mastectomy. Methods All patients who underwent a skin-sparing mastectomy with immediate latissimus dorsi flap breast and NAC reconstruction as a single-stage procedure from 2007 to 2015 were included. Patient demographics, oncologic details, and surgical outcomes were recorded. The BREAST-Q questionnaire was administered to patients to assess the impact and effectiveness of this reconstructive strategy.

Results During the study period, 34 breast and NAC reconstructions in 29 patients were performed at Cork University Hospital. The majority of our patient cohort were non-smokers (93.1\%) and did not receive adjuvant radiotherapy. Postoperative complications were infrequent, with no cases of partial necrosis or complete loss of the nipple. The response rate to the BREAST- 0 was $62 \%(n=18)$. Patients reported high levels of satisfaction with the reconstructed breast $(62 \pm 4)$, nipple reconstruction $(61 \pm 4.8)$, overall outcome $(74.3 \pm 5)$, and psychosocial well-being $(77.7 \pm 3.2)$.

Conclusions Skin-sparing mastectomy with immediate nipple reconstruction during autologous latissimus dorsi reconstruction was demonstrated to be a safe and aesthetically reliable procedure in our cohort, yielding high levels of psychological and physical well-being. A single-stage procedure promotes psychosocial well-being involving issues that are intrinsically linked with breast cancer surgery.

Keywords Breast / Surgery, plastic / Patient satisfaction / Reconstructive surgical procedures

Received: 26 Nov 2017 • Revised: 22 Jul 2018 • Accepted: 2 Oct 2018

pISSN: 2234-6163 • elSSN: 2234-6171 • https://doi.org/10.5999/aps.2017.01725• Arch Plast Surg 2018;45:534-541
Correspondence: Eoin O'Broin Department of Plastic and Reconstructive Surgery, Cork University Hospital, Wilton, Cork, Ireland

Tel: +353-21-454-6400

Fax: +353-21-454-6400

E-mail: eoin.obroin@hse.ie

The data presented in this paper were presented at the Association of Surgeons in Training (ASiT) Conference, Liverpool, UK, March 19, 2016. 


\section{INTRODUCTION}

Skin-sparing mastectomy (SSM), a technique that involves removing all breast and nipple tissue while preserving the native breast envelope, is increasingly offered to women for therapeutic and prophylactic indications [1]. The oncological safety of SSM was initially criticized, but local recurrence rates are similar when SSM is compared with total mastectomy for ductal carcinoma in situ (DCIS) and invasive breast cancer [2,3]. Although initially indicated for DCIS only, SSM has become the standard of care when performing mastectomies with immediate breast reconstruction [4]. Complication rates in patients undergoing SSM and immediate autologous breast reconstruction, even with neoadjuvant chemotherapy or radiotherapy, have been found to be acceptable [5]. Preserving the inframammary fold and breast envelope enables the neoparenchyma to assume the shape of the original breast over time [6]. In addition, the sensation of the skin of the breast is largely retained [7].

For patients undergoing curative mastectomy, the final step in their treatment is nipple-areolar complex (NAC) reconstruction. Traditionally this is performed as a second-stage procedure several months after initial breast reconstruction. This has the benefit of allowing postoperative oedema to diminish, enabling flap stabilization. The recent literature has documented the increasing popularity of immediate nipple reconstructions carried out simultaneously during autologous reconstruction or implant-based reconstruction $[8,9]$. This development has resulted in fewer procedures for the patient, with equivalent aesthetic outcomes [10]. The presence of the nipple on the breast is important for the patient's perception of breast integrity after breast reconstruction [11].

During autologous breast reconstruction with a latissimus dorsi (LD) flap, single-stage nipple reconstruction can be carried out using the skin of the flap. The main benefit of unifying several individual procedures to complete breast reconstruction in a single process is lessening the physical and psychosocial morbidity that was once accepted as an integral part of breast surgery. Whilst a growing body of evidence exists on patient satisfaction with breast reconstruction, few studies have examined the effects of immediate nipple reconstruction. The aim of this study was to evaluate the surgical outcomes and patient satisfaction with immediate breast and nipple reconstruction performed in a single stage after SSM.

\section{METHODS}

\section{Study design and participants}

A prospectively maintained database of all patients undergoing breast reconstruction at Cork University Hospital was used to identify patients suitable for inclusion in this study. Eligible patients were those who underwent SSM with immediate LD flap breast and nipple reconstruction as a single-stage procedure. All patients operated on between September 2007 and September 2015 were included in the study and sent a questionnaire retrospectively in 2015. Hospital In-Patient Enquiry Scheme data was used to ensure adequate capture of the patient cohort by cross-referencing with the database. All patients who underwent this procedure were included in this study, and no exclusion criteria were applied. Ethical approval was obtained from the Clinical Research Ethics Committee at our institution (Ref ECM (qq) 07/07/15).

\section{Surgical technique}

The LD flap was raised in the standard manner, freeing it from its distal attachment, prior to transposing the flap anteriorly. Often, a breast implant was placed over the pectoralis major, beneath the flap to provide more volume and projection to the newly reconstructed breast. The LD flap was then sutured in place. Except for a circle of skin from the LD flap, the remaining skin paddle was de-epithelialized. The circle of skin was sutured to the native skin envelope. The position of the new NAC was well defined by the shape of the skin envelope. The nipple was reconstructed using a C-V flap, utilizing skin from the transposed LD skin paddle at the centre of the circle. The C-V flap offers simple but reliable nipple reconstruction. The C-V flap was designed to be one and a half times the size of the contralateral NAC to allow for expected loss of projection.

\section{Chart review}

Demographic and descriptive patient characteristics recorded included patient age, age at diagnosis, age at reconstruction, smoking status, comorbidities, and BRCA mutation carrier status. The oncologic data collected included histological grade and subtype, estrogen and progesterone receptor status, Her2 status, and the use of neoadjuvant chemotherapy and/or radiotherapy. The following information was extracted about the surgical procedures: oncological surgical procedure, incision type, axillary clearance, weight of resected breast tissue, reconstructive procedure and side, donor site orientation, thoracodorsal nerve resection, division of $\mathrm{LD}$ muscle origin, implant insertion, operative course in hospital, postoperative complications, and disease recurrence.

\section{Patient satisfaction}

A validated patient-reported outcome measures tool was used to assess patient satisfaction with the procedure. The BREAST- 
Qquestionnaire (Memorial Sloan-Kettering Cancer Center and the University of British Columbia, 2006) is a patient-reported outcome instrument designed to evaluate outcomes among women undergoing different types of breast surgery [12]. To evaluate patient satisfaction, the postoperative "Reconstruction" module of the BREAST-Q was used. BREAST-Qquestionnaires were mailed to all study participants with a consent form. Non-responders were reminded with a telephone call at 1 -month and 2-month intervals. Raw responses from the BREAST-Q questionnaire were converted into BREAST-Q scores using the Q-Score program.

\section{Data analysis}

BREAST-Q data were entered into the Q-Score Scoring Software (Memorial Sloan-Kettering Cancer Center, New York, NY, USA) to convert the raw data to the Q-Score. Q-scores range on a scale from 0 to 100 (with a higher number indicating higher satisfaction or quality of life). All patient data were collected and stored anonymously in an encrypted database in Microsoft Excel (Microsoft Corp., Redmond, WA, USA). The database was then imported into GraphPad Prism version 6.0 (GraphPad Software, Inc., La Jolla, CA, USA) for analysis. The statistical analysis was carried out using SPSS version 18 (SPSS Inc., Chicago, IL, USA), with P-values < 0.05 considered to indicate statistical significance.

\section{RESULTS}

\section{Patient demographics}

During the study period, SSM with immediate LD breast and NAC reconstruction was carried out in 34 breasts in 29 patients at our institution. The mean age of the patient cohort was $53.7 \pm 1.9$ years at the time of questionnaire administration, with an average age at reconstruction of $48.0 \pm 1.8$ years (Table 1). The majority of patients were non-smokers (93.1\%). A BRCA mutation was present in $10.3 \%$ of patients $(n=3)$. Neoadjuvant chemotherapy was administered to $17.2 \%$ of patients $(n=5)$, and the majority of patients $(n=17,58.6 \%)$ did not undergo neoadjuvant or adjuvant radiotherapy. The indications

\section{Table 1. Population characteristics}

\begin{tabular}{|lc|}
\hline Characteristics & Value \\
\hline Age of patient cohort (yr) & $53.7 \pm 1.9(38-70)$ \\
Age at diagnosis (yr) & $45.4 \pm 1.6(29-60)$ \\
Age at reconstruction (yr) & $48.0 \pm 1.8(33-68)$ \\
Smokers & $2(6.9)$ \\
BRCA gene mutation carrier & $3(10.3)$ \\
\hline Values are presented as mean \pm standard error of mean (range) or number (\%).
\end{tabular}

for mastectomy were risk reduction $(17.2 \%, \mathrm{n}=5)$, atypical cellular morphology $(3.4 \%, \mathrm{n}=1)$, in situ disease $(51.7 \%, \mathrm{n}=14)$, and invasive carcinoma $(27.6 \%, \mathrm{n}=9)$. There were no bilateral cancer cases.

\section{Operative technique}

All patients underwent SSM, with $17.2 \%(n=5)$ also undergoing axillary clearance. All patients underwent immediate ipsilateral pedicled LD flap reconstruction with C-V flap nipple reconstruction (Table 2). The LD flap was raised in the standard manner. Often, a breast implant was placed in the pre-pectoral space, beneath the flap to provide more volume and projection to the newly reconstructed breast. The LD flap was then sutured in place. Except for a circle of skin from the LD flap, the remaining skin paddle was de-epithelialized. The circle of skin was sutured to the native skin envelope. All risk-reducing mastectomies were bilateral. All reconstructions were performed by the senior author (EOB). In the majority of cases, the mastectomy was performed through a circumareolar incision with lateral extension (Fig. 1). A level II axillary clearance was performed in $17.2 \%(n=5)$ cases.

\section{Postoperative outcomes}

The procedure was well tolerated overall. Patients were reviewed at 1 month, 3 months, 6 months, 9 months, and 12 months postoperatively. Representative pictures of outcomes demonstrate the appearance of the reconstructed breasts at 1 and 9 months postoperatively (Figs. 1 and 2). Nipple projection was

\section{Table 2. Surgical procedures}

\begin{tabular}{|lc|}
\hline Surgical procedures & No. of breasts (\%) \\
\hline Oncological surgical procedure & \\
$\quad$ Skin-sparing mastectomy & $34(100)$ \\
Incision type & \\
$\quad$ Circumareolar with lateral extension & $32(94.1)$ \\
$\quad$ Elliptical & $2(5.9)$ \\
Reconstructive procedure & \\
$\quad$ Immediate LD/NAC reconstruction & $34(100)$ \\
Reconstructed side & \\
Right & $11(32.4)$ \\
Left & $13(38.2)$ \\
$\quad$ Bilateral & $10(29.4)$ \\
Donor site scar orientation & \\
$\quad$ Transverse & $27(79.4)$ \\
Curved oblique & $6(17.7)$ \\
Hybrid incision & $1(2.9)$ \\
Intraoperative procedures & \\
Thoracodorsal nerve resection & $31(91.2)$ \\
Partial division of LD muscle origin & $14(41.2)$ \\
Implant insertion & $32(94.1)$ \\
\hline LD, latissimus dorsi; NAC, nipple-areolar complex. & \\
\hline
\end{tabular}


Fig. 1. A case of immediate NAC reconstruction

A patient aged 48 years who underwent left skin-sparing mastectomy and immediate latissimus dorsi breast and nipple-areolar complex (NAC) reconstruction, and results at 1 month. Preoperative $(A, C, E)$ and postoperative images $(B, D, F)$. She reported very high satisfaction with the overall outcome and with her breasts, with scores of 100 and 100, respectively.
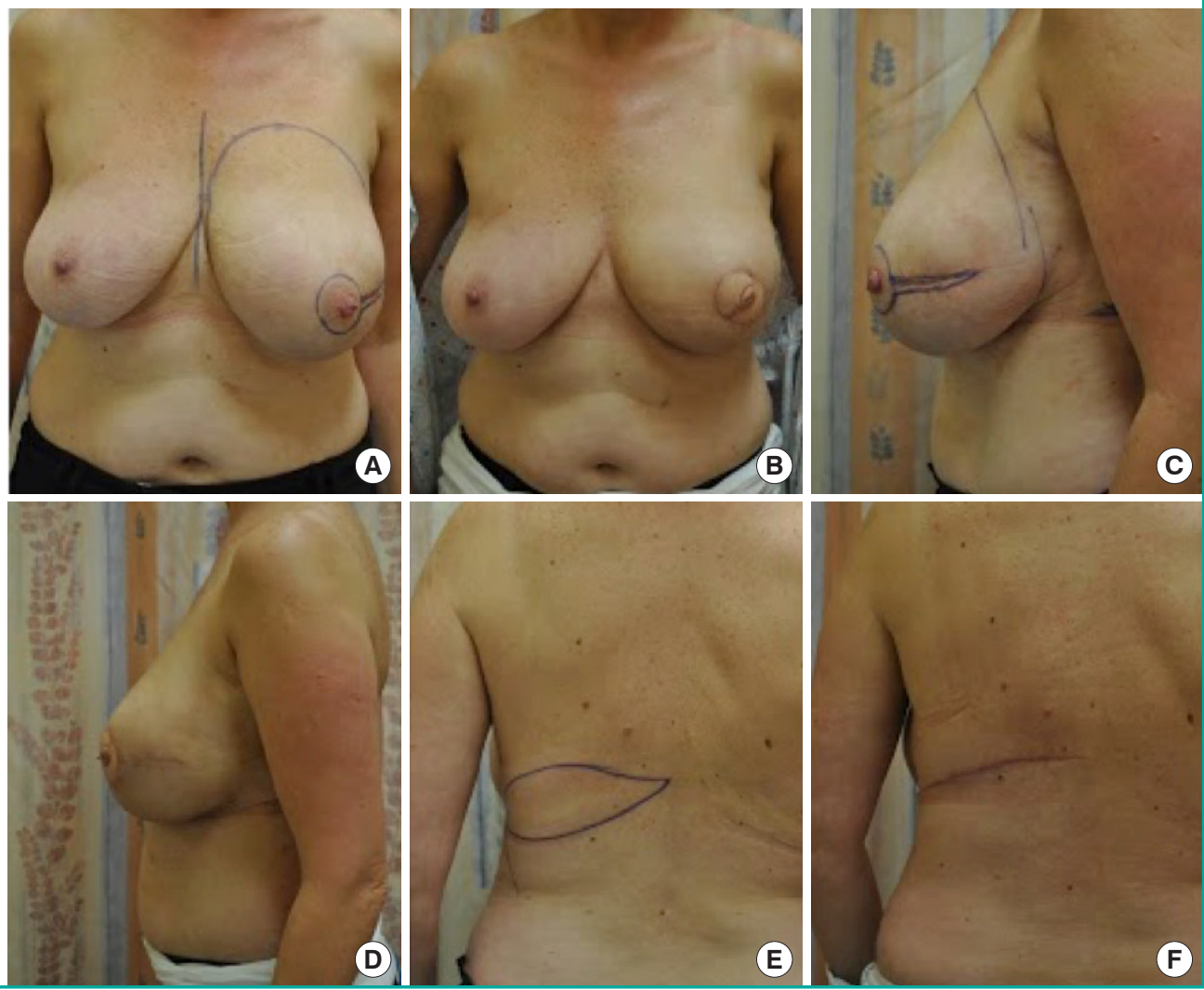

(E)

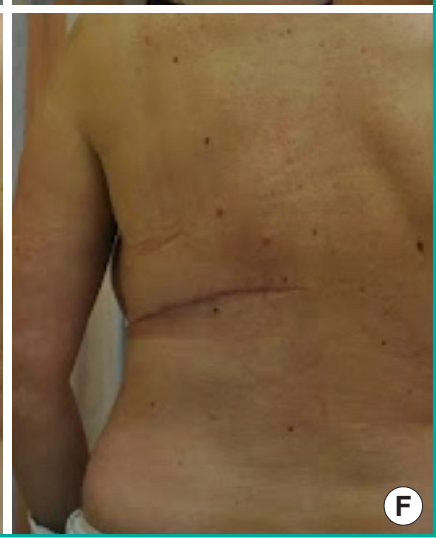

Fig. 2. A case of right immediate NAC reconstruction

A patient aged 53 years who underwent right skin-sparing mastectomy and immediate latissimus dorsi breast and nipple-areolar complex (NAC) reconstruction, and results at 9 months. Preoperative $(A, C, E)$ and postoperative images $(B, D, F)$.
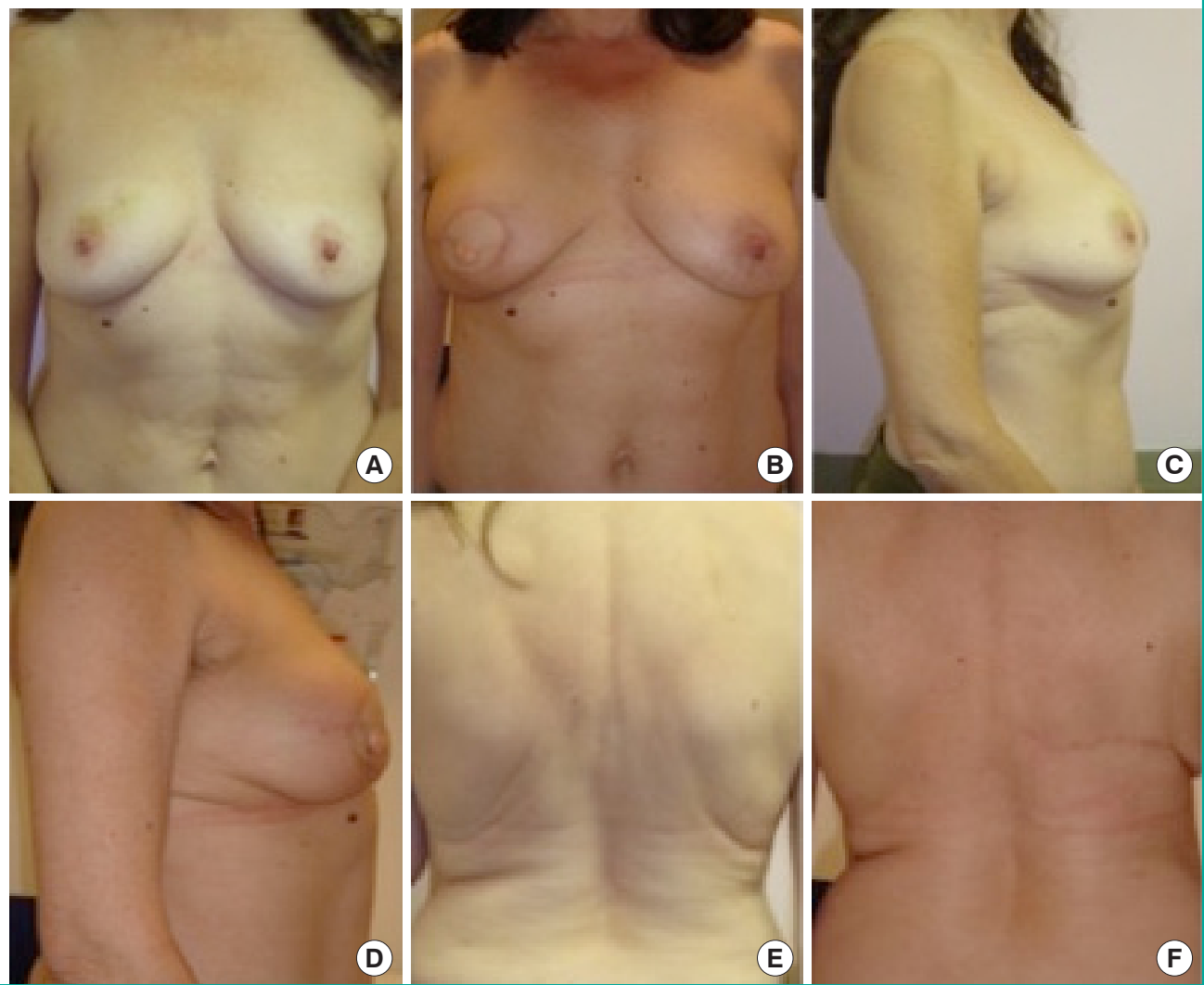


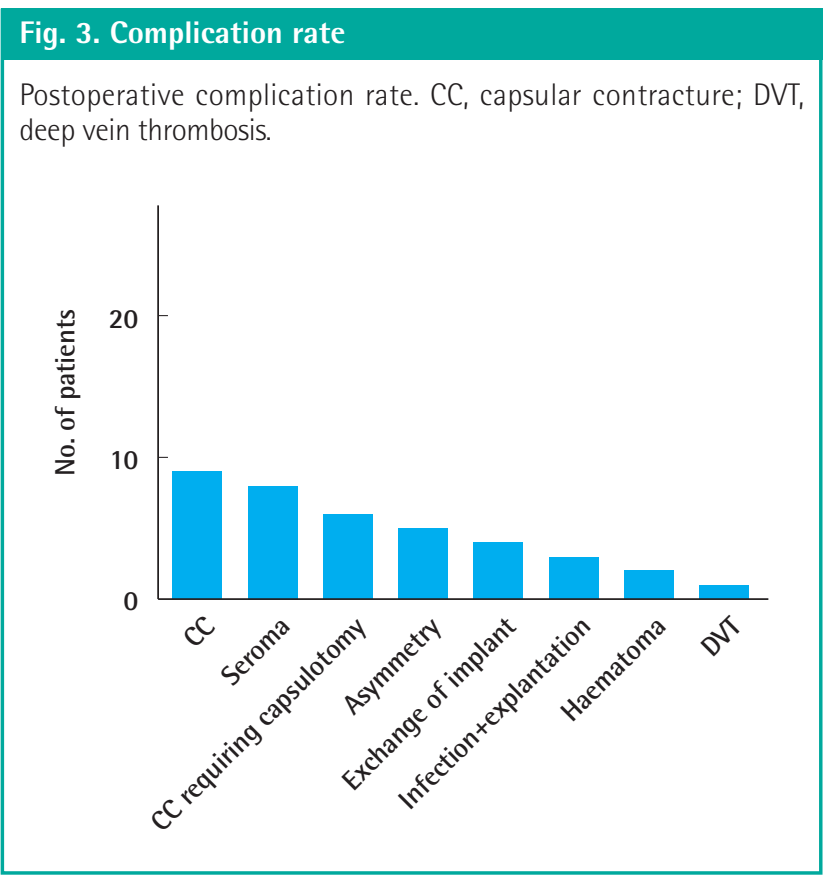

documented in $66 \%$ of patients $(n=19)$. The mean nipple projection at 1 year was $6 \mathrm{~mm}$. Capsular contracture $(31.0 \%, \mathrm{n}=9)$ was the most frequently encountered complication (Fig. 3). At 1 year, two patients had grade I contracture, three patients had grade II contracture, and four patients had grade III contracture. A seroma formed postoperatively in $27.5 \%$ of patients $(n=8)$ (Fig. 3). These were all treated in outpatient department office visits at 2,4 , and 8 weeks. The mean volume of fluid recorded was $70 \pm 10.3 \mathrm{~mL}$ (range, 30-100 mL). Three cases of infection were reported, all of which required removal of the implant. There were no cases of partial necrosis or complete loss of the nipple. Disease progression within 5 years of surgery occurred in $13.8 \%$ of patients $(n=4)$. Interestingly, NAC tattooing, which usually takes place approximately 3 months postoperatively, was performed in only $6.9 \%$ of patients $(n=2)$. No patients underwent symmetrising surgery postoperatively.

\section{Patient satisfaction}

The response rate to the BREAST-Q was $62 \%(n=18)$. The mean time from surgery to patient questionnaire completion was 3.4 years. The overall physical well-being scores demonstrated that the procedure was associated with a high level of satisfaction. Of note, many patients complained of upper back pain and discomfort in the breast area following surgery $(n=4)$. The Q-score was used to assess patient-reported outcomes in a standardized manner. The highest patient-reported satisfaction score was for the surgical staff $(95.8 \pm 2.3)$ and information received $(79.5 \pm 3.5)$ (Table 3 ). Patients reported a high level of satisfaction with the reconstructed breast $(62 \pm 4)$, physical

\section{Table 3. Values of $\mathbf{0}$-Scores}

\begin{tabular}{|lc|}
\hline Q-Scores & Value \\
\hline Satisfaction & \\
Satisfaction with overall outcome & $74.3 \pm 5.5(32-100)$ \\
Satisfaction with breasts & $62.0 \pm 4.0(32-100)$ \\
Satisfaction with nipples & $61.0 \pm 4.8(26-100)$ \\
Well-being & \\
Psychosocial well-being & $77.7 \pm 3.2(49-100)$ \\
Sexual well-being & $57.0 \pm 2.7(41-83)$ \\
Physical well-being: chest & $80.8 \pm 2.6(57-91)$ \\
Satisfaction with care and expectations & \\
Satisfaction with information & $79.5 \pm 3.2(53-100)$ \\
Satisfaction with surgeon & $95.8 \pm 2.3(66-100)$ \\
Satisfaction with medical staff & $95.8 \pm 2.4(59-100)$ \\
Satisfaction with office staff & $94.9 \pm 3.0(58-100)$ \\
\hline Values are presented as mean \pm standard error of mean (range).
\end{tabular}

well-being (80.8 \pm 2.6$)$, and the overall outcome $(74.3 \pm 5)$. Psychosocial well-being was also rated highly $(77.7 \pm 3.2)$. Good satisfaction ratings were also demonstrated for the appearance of the nipple $(61 \pm 4.8)$ and sexual well-being $(57 \pm$ 2.6). There was no statistically significant difference in the $\mathrm{Q}$ scores for the overall outcome, breast outcome, and nipple outcome among patients with significant postoperative complications and those without such complications. Patient satisfaction with the overall outcome was not impacted by smoking status $(P=0.031)$, age $(P=0.023)$, or. interestingly, chest wall radiation $(\mathrm{P}=0.034)$.

\section{DISCUSSION}

SSM is widely performed in patients who receive an early diagnosis of early breast cancer, and there is good evidence supporting its oncologic safety compared with total mastectomy [13]. The aim of reconstructive breast surgery is to recreate the breast mound and NAC with a technique that is both predictable and aesthetically pleasing. Without a NAC, breast mound reconstruction may not appear visually complete. Traditionally, NAC reconstruction has been carried out as a secondary procedure. However, recent literature has provided support for immediate NAC reconstruction as a single-stage procedure.

Immediate NAC reconstruction using an LD flap in SSM is a cost-effective method that adds 15 minutes of operative time to the surgical procedure. Our rates of seroma formation (27.5\%) and postoperative infection (10.3\%) were in keeping with the reported literature. The rate of capsular contracture in our cohort was high at 1 year postoperatively. Capsular contracture occurs more frequently after breast reconstruction than after aesthetic augmentation. A factor that may have influenced its oc- 
currence in our study was the high rate of pre-operative and postoperative exposure to chemotherapy. This impairs the local immunologic status of tissues and increases the rate of subclinical infection [14]. Delay et al. [15] described a similar technique using an LD flap in a smaller cohort with similar postoperative results. Alternative autologous techniques that have been described include the muscle-sparing transverse rectus abdominis muscle (TRAM) flap and the deep inferior epigastric perforator flap $[16,17]$. The majority of our patient cohort were nonsmokers (93.1\%) and did not receive adjuvant radiotherapy. This contributed to our low complication rate and highlights the importance of patient selection for this technique. The healing period of the nipple does not exceed that of the breast and back. Some institutions reserve the combination of autologous reconstruction and immediate NAC reconstruction for patients who are not being considered for radiotherapy [18].

The main argument for delaying NAC reconstruction is centred on the challenge of determining the correct NAC position. However, following SSM, the only visible part of the LD skin paddle is the small circle that occupies the position of the original NAC. Therefore, creation of the reconstructed nipple at the centre of this circle using the skin from the LD skin paddle ensures that it is situated at a position most similar to its pre-operative location. Our cohort had no cases of nipple reconstruction complicated by partial or full necrosis. Postoperative NAC tattooing uptake was quite infrequent at our institution (6.9\%). The reason for such low uptake is currently being explored, but we advise patients of its aesthetic benefits. Patient satisfaction with NAC tattooing has been reported to be as high as $84 \%$ [19]. Further efforts will be made in the future to translate this to our patient cohort.

All nipple reconstructions in this study were performed using a C-V flap, utilizing skin from the transposed LD skin paddle at the centre of the circle. This is the senior author's preferred technique. Alternative techniques for simultaneous NAC reconstruction include a modified star flap or the Hartrampf wraparound flap described by Hyza et al. [18]. Williams et al. [10] introduced a further variation, involving TRAM flap breast reconstruction with simultaneous NAC reconstruction using a fishtail or trilobed flap in 10 patients. The C-V flap can result in up to a $50 \%$ loss in projection over time due to absorption of the central fat core [20]. Our technique allows for expected loss of projection by designing the flap to be one and a half times the size of the contralateral NAC. Hong et al. [9] and Williams et al. [10] objectively described aesthetic nipple projections and symmetry at 1 year postoperatively. Similarly, Delay et al. [15] appropriately described the objective and subjective assessment of nipple projection at 2 years postoperatively, with a mean re- sidual projection of $6.8 \mathrm{~mm}$ after 2 years, in keeping with our results.

Conservative mastectomy procedures (i.e., SSM and nipplesparing mastectomy [NSM]), are relatively new surgical approaches to breast cancer surgery. The paradigm shift towards NSM followed low satisfaction rates with the appearance and sensitivity of the reconstructed nipple [21]. However, a recent study by van Verschuer et al. [22] comparing SSM and NSM showed satisfaction with breasts and the overall outcome to be higher in patients who underwent SSM. Furthermore, a Swedish prospective study found total loss of touch sensation in the nipple in $62 \%$ of patients following NSM [7]. Our patients rated the overall outcomes of the procedure highly (at 74.3), with modest satisfaction with the overall outcome of both breast and nipple reconstruction. We feel that appropriate pre-operative counselling contributed to these high patient satisfaction rates by involving patients in the decision-making process. This is achieved by using a summary of the experiences of other women with the aid of previous postoperative photography. The high satisfaction of expectations was reflected in the high scores for satisfaction with the consultant surgeon, breast team, nursing staff, and office staff.

Outcomes in emotional, physical and sexual well-being reflect pre-existing issues that are intrinsically linked to the diagnosis and treatment for breast cancer. In our study, psychosocial and physical well-being were equally rated very highly. This may reflect the possible role of the NAC in the assimilation of the new breast image into the body image of the woman. This is also observed by Delay et al. [15], where $86.6 \%$ of patients reported that immediate nipple reconstruction was "very important" and 13.4\% considered it "important." Jabor et al. [23] found that patients who had a longer time interval between breast mound and NAC reconstruction reported poorer overall levels of satisfaction. We expected that sexual well-being would be the lowestrated domain that we evaluated. It was the most often skipped subtheme on the questionnaire, and is likely an issue that cannot be dealt with by the surgical team [24]. It is, nonetheless, a very important domain of quality of life and should be examined.

The authors acknowledge some limitations of this study. The time of the surgical procedure was not matched to the time of BREAST-Q completion, which represents a source of potential bias. Nipple projection and maintenance of nipple projection over time were not objectively assessed given the retrospective nature of the study. Loss of nipple projection is a risk with all reconstructions and has been reported to be as high as $40 \%$ in the literature (for nipple reconstruction using the C-V flap technique) [20]. However, loss of nipple projection has been shown to have a minimal impact on overall patient satisfaction [25]. 
Furthermore, our study reflects an unmatched cohort. No study has yet been conducted of delayed nipple reconstruction using the BREAST-Q tool. Despite this, there is a growing body of literature describing the benefits of immediate NAC over traditional 2-stage reconstruction, from both aesthetic and psychological perspectives.

In conclusion, SSM with immediate nipple reconstruction during autologous $\mathrm{LD}$ reconstruction was demonstrated to be a safe and aesthetically reliable procedure in our cohort, yielding high levels of psychological and physical well-being. Reconstructions may be completed sooner and with fewer procedures. A single-stage procedure promotes psychosocial well-being involving issues that are intrinsically linked with breast cancer surgery.

\section{NOTES}

\section{Conflict of interest}

No potential conflict of interest relevant to this article was reported.

\section{Ethical approval}

The study was approved by the Institutional Review Board of Cork University Hospital (Ref ECM (qq) 07/07/15) and performed in accordance with the principles of the Declaration of Helsinki. Written informed consents were obtained.

\section{Patient consent}

The patients provided written informed consent for the publication and the use of their images.

\section{REFERENCES}

1. Warren Peled A, Foster RD, Stover AC, et al. Outcomes after total skin-sparing mastectomy and immediate reconstruction in 657 breasts. Ann Surg Oncol 2012;19:3402-9.

2. Carlson GW, Losken A, Moore B, et al. Results of immediate breast reconstruction after skin-sparing mastectomy. Ann Plast Surg 2001;46:222-8.

3. Romics L Jr, Chew BK, Weiler-Mithoff E, et al. Ten-year follow-up of skin-sparing mastectomy followed by immediate breast reconstruction. Br J Surg 2012;99:799-806.

4. Carlson GW, Bostwick J 3rd, Styblo TM, et al. Skin-sparing mastectomy: oncologic and reconstructive considerations. Ann Surg 1997;225:570-5.

5. Zinzindohoue C, Bertrand P, Michel A, et al. A prospective study on skin-sparing mastectomy for immediate breast reconstruction with latissimus dorsi flap after neoadjuvant chemotherapy and radiotherapy in invasive breast carcinoma. Ann Surg Oncol 2016;23:2350-6.

6. Davies K, Allan L, Roblin P, et al. Factors affecting post-operative complications following skin sparing mastectomy with immediate breast reconstruction. Breast 2011;20:21-5.

7. Gahm J, Hansson P, Brandberg Y, et al. Breast sensibility after bilateral risk-reducing mastectomy and immediate breast reconstruction: a prospective study. J Plast Reconstr Aesthet Surg 2013;66:1521-7.

8. Craig ES, Walker ME, Salomon J, et al. Immediate nipple reconstruction utilizing the DIEP flap in areola-sparing mastectomy. Microsurgery 2013;33:125-9.

9. Hong KY, Kim YE, Minn KW, et al. Immediate nipple reconstruction during implant-based breast reconstruction. Aesthetic Plast Surg 2017;41:793-9.

10. Williams EH, Rosenberg LZ, Kolm P, et al. Immediate nipple reconstruction on a free TRAM flap breast reconstruction. Plast Reconstr Surg 2007;120:1115-24.

11. Wellisch DK, Schain WS, Noone RB, et al. The psychological contribution of nipple addition in breast reconstruction. Plast Reconstr Surg 1987;80:699-704.

12. Pusic AL, Klassen AF, Scott AM, et al. Development of a new patient-reported outcome measure for breast surgery: the BREAST-Q. Plast Reconstr Surg 2009; 124:345-53.

13. Toth BA, Forley BG, Calabria R. Retrospective study of the skin-sparing mastectomy in breast reconstruction. Plast Reconstr Surg 1999;104:77-84.

14. Taylor CW, Horgan K, Dodwell D. Oncological aspects of breast reconstruction. Breast 2005; 14:118-30.

15. Delay E, Mojallal A, Vasseur C, et al. Immediate nipple reconstruction during immediate autologous latissimus breast reconstruction. Plast Reconstr Surg 2006;118:1303-12.

16. Kim HR, Lim JS, Kim SM, et al. One-stage nipple and breast reconstruction following areola-sparing mastectomy. Arch Plast Surg 2013;40:553-8.

17. Korn PT, Maia M, Chen MH. Immediate nipple reconstruction with autologous breast reconstruction following areolasparing mastectomy: a marriage of aesthetics and oncologic principles. Plast Reconstr Surg 2014;134(4 Suppl 1):79-80.

18. Hyza P, Streit L, Vesely J, et al. New technique of immediate nipple reconstruction during immediate autologous DIEP or MS-TRAM breast reconstruction. Ann Plast Surg 2015; 74:645-51.

19. Spear SL, Arias J. Long-term experience with nipple-areola tattooing. Ann Plast Surg 1995;35:232-6.

20. Jalini L, Lund J, Kurup V. Nipple reconstruction using the C-V flap technique: long-term outcomes and patient satisfaction. World J Plast Surg 2017;6:68-73. 
21. Galimberti V, Vicini E, Corso G, et al. Nipple-sparing and skin-sparing mastectomy: review of aims, oncological safety and contraindications. Breast 2017;34 Suppl 1:S82-4.

22. van Verschuer VM, Mureau MA, Gopie JP, et al. Patient satisfaction and nipple-areola sensitivity after bilateral prophylactic mastectomy and immediate implant breast reconstruction in a high breast cancer risk population: nipplesparing mastectomy versus skin-sparing mastectomy. Ann Plast Surg 2016;77:145-52.
23. Jabor MA, Shayani P, Collins DR Jr, et al. Nipple-areola reconstruction: satisfaction and clinical determinants. Plast Reconstr Surg 2002;110:457-63.

24. Jeevan R, Cromwell D, Browne J, et al. National mastectomy and breast reconstruction audit 2011. Leeds: NHS Information Centre, 2011.

25. Sisti A, Grimaldi L, Tassinari J, et al. Nipple-areola complex reconstruction techniques: a literature review. Eur J Surg Oncol 2016;42:441-65. 